\section{A randomized clinical trial comparing fixed vs pro-re-nata dosing of Ozurdex in refractory diabetic macular oedema (OZDRY study)}

J Ramu1', Y Yang², G Menon ${ }^{3}$, C Bailey4, N Narendran'2, C Bunce ${ }^{1}$, A Quartilho ${ }^{1}$, AT Prevost ${ }^{5}$, P Hykin $^{1}$ and S Sivaprasad ${ }^{1}$ for the OZDRY Study Group

\begin{abstract}
Objective To compare the clinical effectiveness and safety of 5-monthly fixed dosing $v$ s pro-re-nata (PRN) Ozurdex treatment in patients with refractory diabetic macular oedema (DMO).

Design Prospective, multicentre, randomized active-controlled non-inferiority clinical trial. Participants Participants were $\mathbf{1 0 0}$ patients who attended Medical Retina Clinics for management of centre-involving refractory DMO.

Interventions Participants were randomized $1: 1$ to either 5-monthly fixed dosing or optical coherence tomography (OCT)-guided PRN regimen of Ozurdex therapy for DMO. Data were collected on best-corrected visual acuity (BCVA), patient-reported outcome measures (PROM), macular thickness and morphology, diabetic retinopathy status, number of injections and adverse events from baseline for a period of $\mathbf{1 2}$ months. Main outcome measures The primary outcome was the difference between arms in change in BCVA from baseline to 12 months. The prespecified non-inferiority margin was five ETDRS letters. Key secondary outcomes included change in PROM scores, change in macular thickness, change in retinopathy and macular morphology, and safety profile. Results The mean change in BCVA was +1.48 (SD 14.8) in the fixed arm $v s-0.17$ (SD 13.1) in the PRN arm, with adjusted effect estimate $+0.97,90 \%$ confidence interval $(-4.01,+5.95), P=0.02$ (per protocol analysis). The conclusions of the ITT analysis were primarily supportive, $-0.34(-5.49,4.81)$ $P=0.07$, but sensitive to an alternative
\end{abstract}

assumption on missing data, $+0.28(-4.72$, 5.27) $P=0.04$.

Conclusions The mean change in BCVA with 5-monthly fixed dosing of Ozurdex was non-inferior to OCT-guided PRN Ozurdex therapy for refractory DMO based on a per protocol analysis.

Eye (2015) 29, 1603-1612; doi:10.1038/eye.2015.214; published online 23 October 2015

\section{Introduction}

Centre-involving diabetic macular oedema (DMO) is a leading cause of moderate visual loss in diabetes. ${ }^{1}$ The visual outcome and visionrelated quality of life of people with centreinvolving DMO have significantly improved with the initiation of inhibitors of vascular endothelial growth factor (VEGF). ${ }^{2,3}$ However, many patients still need frequent and multiple injections of antiVEGF and up to $50 \%$ of treated patients do not achieve long-term resolution of $\mathrm{DMO}^{4,5}$

Therefore, there is a significant unmet need for alternative interventions for refractory DMO. ${ }^{6}$

Intravitreal steroids were the first class of intravitreal drugs that were evaluated for the treatment of this condition and remain a promising treatment modality for people with DMO owing to both its anti-inflammatory and antivascular permeability effects. ${ }^{7,8}$ The Ozurdex (Allergan Inc.) drug delivery system is a sustained-release formulation for posteriorsegment delivery of $700 \mu \mathrm{g}$ dexamethasone. The Phase 3 MEAD study that evaluated the role of six monthly pro-re-nata (PRN) dosing of Ozurdex for DMO reported that $22 \%$ of patients improved $\geq 15$ Early Treatment Diabetic
${ }^{1}$ NIHR Moorfields Biomedical Research Centre, London, UK

${ }^{2}$ The Royal Wolverhampton NHS Trust, Birmingham, UK

${ }^{3}$ Frimley Health NHS Foundation Trust, Surrey, UK

${ }^{4}$ Bristol Eye Hospital, Bristol, UK

${ }^{5}$ Division of Health and Social Care Research, King's College London London, UK

Correspondence: S Sivaprasad, NIHR Moorfields Biomedical Research Centre, London EC1V 2PD, UK Tel: +44 (0)78 1788 6759; Fax: +44 (0)20 84913978. E-mail: senswathi@aol.com

Received: 14 September 2015

Accepted in revised form:

1 October 2015

Published online:

23 October 2015 
Retinopathy Study (ETDRS) letters at the end of 3 years. ${ }^{9}$ Another trial that compared the combination of Ozurdex and laser therapy $v$ s laser therapy (PLACID) reported that, to obtain a sustained effect of Ozurdex, the treatment should be repeated at shorter intervals than every 6 months based on the changes observed in macular thickness on optical coherence tomography (OCT) and visual acuity. ${ }^{10}$ The OCTOME study reported that the maximum treatment response of the drug occurred at 12 weeks before the effect wore off gradually. Therefore, a more frequent dosing between 16 and 20 weeks may be necessary to avoid the undulating effects on macular thickness and visual acuity. ${ }^{11}$ A 16-weekly PRN dosing evaluated in the BEVORDEX study reported that $41 \%$ of the patients in the study improved $\geq 10$ letters. ${ }^{12}$ Therefore, a great deal of uncertainty still exists on the optimal dosing of Ozurdex to adopt for patients with DMO.

The objective of this study was to compare the riskbenefit ratio of 5-monthly fixed dosing vs OCT-guided PRN dosing of Ozurdex in centre-involving refractory DMO. The PRN dosing was designed to mirror the treatment protocol of Ozurdex in the NHS. Patients can be injected as early as 4 months, so this pathway was planned to ensure that patients receive Ozurdex at least 4 months after last dosing if deemed eligible based on retreatment criteria. The fixed arm was the investigational arm where we wanted to explore whether patients could be observed every 5 months without affecting the efficacy and safety profile.

The primary objective was to evaluate whether 5-monthly fixed dosing of $700 \mu \mathrm{g}$ Ozurdex is non-inferior to OCT-guided PRN dosing in patients with DMO. Our null hypothesis was that the change in best-corrected visual acuity (BCVA) between baseline and 12 months is $>5$ ETDRS letters lower in the fixed dosing (investigative) arm than in the OCT-guided PRN dosing (standard) arm, to be assessed after adjusting for baseline BCVA and study site.

\section{Methods \\ Study design}

This is a multicentre, prospective, randomized, activecontrolled, non-inferiority study conducted across five sites in the United Kingdom. The study was registered at www.clinicaltrials.gov/NCT01892163. The study protocol was approved by the UK Collaborative Research Ethics Committee (12/LO/1534). The principles of Good Clinical Practice were adhered throughout in accordance with the Declaration of Helsinki. The trial was registered.

\section{Study population}

Eligible patients were at least 18 years old with type 1 or type 2 diabetes. The key eligibility criteria for the study eye included: (1) best-corrected ETDRS visual acuity letter score 73-34 (20/40-20/200), (2) definite retinal thickening owing to DMO on clinical examination involving the centre of the macula assessed to be the main cause of visual loss, and (3) retinal thickness measured on spectral domain OCT $>300 \mu \mathrm{m}$ in the central subfield (CST) despite treatment. Principal exclusion criteria included: (1) macular ischaemia defined as angiographic evidence of foveal avascular zone of $>1000 \mu \mathrm{m}$ in diameter or the presence of severe perifoveal intercapillary loss,

(2) previous treatment for $\mathrm{DMO}$ with intravitreal or peribulbar steroids in the past 6 months; anti-VEGF therapy in the last 1 month or macular laser within the prior 3 months, (3) active proliferative diabetic retinopathy requiring treatment at screening, (4) substantial cataract that, in the opinion of the investigator, was likely to be decreasing visual acuity by $\geq 3$ lines (ie, cataract would be reducing acuity to $20 / 40$ or worse if the eye was otherwise normal), (5) vitrectomised eye, (6) a diagnosis of glaucoma that, in the opinion of a glaucoma specialist, was at high risk of progression or ocular hypertension requiring at least one topical medication, and (7) coexistent disease affecting the visual acuity of the study eye.

One eye was selected and treated as the study eye. If both eyes were eligible, the eye with the better visual acuity at screening was selected for treatment, unless the patient preferred otherwise.

\section{Interventions}

All patients received baseline Ozurdex injection. Intravitreal Ozurdex injections were performed under local anaesthesia and postinjection topical antibiotics were used. Further Ozurdex injections in each arm were performed according to protocol-defined retreatment criteria. In the intervention arm (fixed dosing), mandated intravitreal Ozurdex was given at baseline, 5, and 10 months if the criteria for deferred treatment were not met at those time points. In the standard arm, participants were observed at baseline, 4 months, and then monthly to assess the need for re-treatment. If the participants in standard arm were re-treated at any point, the next visit was after 4 months. In addition, safety visits were carried out at 1 and 8 weeks after any Ozurdex injection in either treatment arms. If there was any safety concern in the opinion of the investigator, more frequent optional postinjection assessment visits were allowed.

Re-treatment with Ozurdex was indicated if the CST on OCT exceeded $300 \mu \mathrm{m}$ and the intraocular pressure (IOP) was $\leq 25 \mathrm{~mm} \mathrm{Hg}$. If the IOP was between 26 and 
$30 \mathrm{~mm} \mathrm{Hg}$, topical antiglaucoma eye drops were given before treatment with Ozurdex at the same sitting. If the IOP recorded was $\geq 30 \mathrm{~mm} \mathrm{Hg}$, antiglaucoma eye drops were given and the patient was reviewed a week later and Ozurdex was injected only if the IOP had reduced to $<30 \mathrm{~mm} \mathrm{Hg}$. Ozurdex treatment was deferred if the BCVA was better than 83 letters or the IOP was $\geq 30 \mathrm{~mm} \mathrm{Hg}$ while on Ozurdex therapy or there was evidence of intraocular infection or severe inflammation. The total duration of study participation was 12 months.

\section{Randomization and treatment allocation}

The study patients were randomized using a $1: 1$ allocation ratio into either the fixed dosing or the PRN dosing schedule of Ozurdex therapy via a bespoke web-based randomization system hosted at the King's Clinical Trials Unit using the randomization sequence generation of block randomization with randomly varying block sizes. The use of concealed randomly varying block sizes ensured that treatment allocation did not become predictably determined towards the end of each block and thus protected prerandomization allocation concealment.

\section{Masking}

The primary outcome assessors (optometrists and OCT technicians) at each site were masked to treatment allocation. The clinicians who administered the study treatment and those who performed the safety evaluations were not masked to the treatment arms.

\section{Efficacy and safety assessments}

We assessed BCVA using ETDRS charts at a starting distance of $4 \mathrm{~m}$. The PROM was assessed using the visionspecific National Eye Institute Visual Function Questionnaire $^{13}$ and the validated diabetic retinopathy specific questionnaire on quality of life ${ }^{14}$ administered by staff at each site at baseline and 12 months. In addition, all patients completed a diabetic Retinopathy-specific Treatment Satisfaction Questionnaire ${ }^{15}$ at baseline and at 12 months.

The OCT technicians performed OCT at every study visit using spectral domain OCT (Spectralis OCT, Heidelberg Engineering, Heidelberg, Germany). The CST was obtained directly from the ETDRS map on the OCT. The morphological characteristics were graded by individual site investigators. Autofluorescence of the macula was performed on the Spectralis. The total area of hyperautofluorescence and hypoautofluorescence were measured using the in-built measuring tool. Fluorescein angiography was performed at baseline and month 12 . In addition, red-free and four-field colour photographic images of the retina of the study eye were performed before fluorescein angiography at baseline and 12 months. The greatest linear diameter and area of foveal avascular zone were recorded by site investigators. The macular oedema was graded as predominantly focal or diffuse. The diabetic retinopathy grades were classified as mild, moderate, severe non-proliferative diabetic retinopathy, and treated proliferative diabetic retinopathy according to the International Clinical Disease Severity Scale. ${ }^{16}$ The investigators also recorded the presence or absence of hard exudates in the central $6 \mathrm{~mm}$ of the retina.

\section{Safety assessments}

Safety was assessed by the 12-month incidence of adverse events (AEs) and serious AEs by ophthalmic examinations and IOP measurements over the 12-month assessment period. IOP was measured at each visit. The presence and severity of lens opacities were measured during slit-lamp examination using standardized photographs and the Lens Opacities Classification System II. ${ }^{17}$ Systemic blood pressure and glycated haemoglobin (HbA1C) levels were also measured at baseline and 12 months.

\section{Concomitant procedures}

All medication(s)/treatment(s) except intravitreal antiVEGF, periocular, and intravitreal steroids and macular laser treatment were permitted during the trial period in the study eye of the patients. IOP-lowering agents or surgery were allowed and consultations with a glaucoma specialist were permitted. Cataract surgery for visually significant cataract during the study period was at the discretion of the investigator. A masked grader determined whether the cataract was visually significant before planned cataract surgery. Pan retinal photocoagulation for retinal neovascularisation in both the study and non-study eye was also permitted.

\section{Outcome measures}

The primary outcome was assessed as the difference between arms in the mean change in BCVA between baseline and 12 months. Secondary outcomes included categorical outcomes in a gain or loss in visual acuity, differences between arms in patient-related outcome scores, number of injections, change in central subfield thickness and morphological characteristics of the macula on OCT, autofluorescence, change in grading of diabetic retinopathy, the greatest diameter of the foveal avascular zone on fluorescein angiography, and AEs. 


\section{Sample size}

This study was designed as a non-inferiority trial with the non-inferiority limit for the difference between study arms in the mean change in visual acuity at 12 months of 5 ETDRS letters lower under fixed dosing, assessed after adjusting for baseline BCVA ETDRS letter score and study site. If there is no statistically significant difference in the change in BCVA ETDRS letter score between baseline and 12 months in the populations represented by two study arms, a sample size of 90 patients was required to be $83 \%$ certain that the lower limit of a one-sided 95\% confidence interval (or equivalently a $90 \%$ two-sided confidence interval) would be above the non-inferiority limit of 5 letters, assuming that the common SD was 9 letters. The SD is based on the results of the Ranibizumab (RESOLVE) study. ${ }^{18}$ The non-inferiority margin of five ETDRS letters is based on the CATT study (in which it is recognised as a commonly accepted margin) ${ }^{19}$ and the results of the PLACID study. ${ }^{10}$ Allowing for $10 \%$ missing data, 100 patients were randomized (ie, 50 patients per study arm).

\section{Statistical analysis}

For the non-inferiority analysis of the primary outcome, the following two populations were predefined: Intention to treat (ITT: all patients randomized) and per protocol (PP: those who met with the eligibility criteria and received the randomized treatment in accordance with the protocol). Corresponding ITT and PP 'available case' sample populations were predefined as those cases with available primary outcome data. Three patients did not provide primary outcome data at 12 months, one in the fixed arm and two in the PRN arm. This was less than the proportion anticipated to be lost to follow-up (10\%) confirming the predefined available case analysis approach to provide valid treatment effect estimates. At the request of the Data Monitoring Committee, an additional post-hoc sensitivity analysis with alternative missing data assumptions was then conducted for the ITT population. This used in place of available case analysis a last observation carried forward (LOCF) analysis approach, which carried forward data in these three patients who did not provide primary outcome data at 12 months.

The following significance levels were predefined. The primary outcome analysis used a one-sided $P$-value of 0.05 , with a one-sided $95 \%$ confidence interval (or equivalently a two-sided $90 \%$ confidence interval), in accordance with a non-inferiority design. All other statistical tests used a two-sided $P$-value of 0.05 , with a two-sided 95\% confidence interval.

Summary measures for the baseline characteristics of each arm are presented as mean and SD for continuous (approximate) normally distributed variables, medians and interquartile ranges for non-normally distributed variables, and frequencies and percentages for categorical variables. Treatment effect estimates are reported as differences in means for continuous (approximate) normal data, differences in medians for non-normally distributed data, and as odds ratios (using logistic regression) for binary data, after adjusting for baseline BCVA, study site, and the respective baseline covariate, where available. Effect estimates are presented with a two-sided 95\% confidence interval.

A predefined sensitivity analysis was conducted to assess the effect of having cataract surgery during the study on the primary outcome. This was restricted to those included in the primary analysis and was carried out by replacing the final visual acuity measurement with the last available visual acuity measurement before surgery and repeating the primary analysis.

A related within-subgroup analysis of the primary outcome was performed on patients who were pseudophakic at baseline. This provided an unbiased but less precise estimate of the treatment effect in this subgroup, which is free from any cataract-related issues. Secondary outcomes were analysed using ITT analysis to compare arms.

Except for the post-hoc ITT using LOCF, all analyses were prespecified and detailed in a Statistical Analysis Plan approved prior to data lock and therefore prior to any analyses and treatment allocation unmasking. All statistical analyses were conducted using Stata/IC (version 13.1, Stata Corp., College Station, TX, USA).

\section{Results}

A total of 100 patients were enrolled from February 2013 to November 2014 and randomized to study treatment across five sites. Figure 1 shows the CONSORT diagram that describes the flow of participants at each stage. Tables 1 and 2 shows that the treatment arms were similar at baseline with respect to the demographics and study eye characteristics.

\section{Primary outcome}

The ITT analysis effect estimate was $-0.34(-5.49,4.81)$. Although this available case analysis interval overlapped the non-inferiority margin by half a letter, this was not observed in either PP analysis or the post-hoc ITT sensitivity analysis based on LOCF. For the ITT (available case), the mean improvement in the visual acuity letter score in the fixed arm was 0.53 letters and 0 in the PRN arm. Both the PP analysis effect estimate of $0.97,90 \%$ CI $(-4.01,5.95)$ and the post-hoc ITT sensitivity analysis effect estimate of $0.28,90 \% \mathrm{CI}(-4.72,5.27)$ support the claim of 


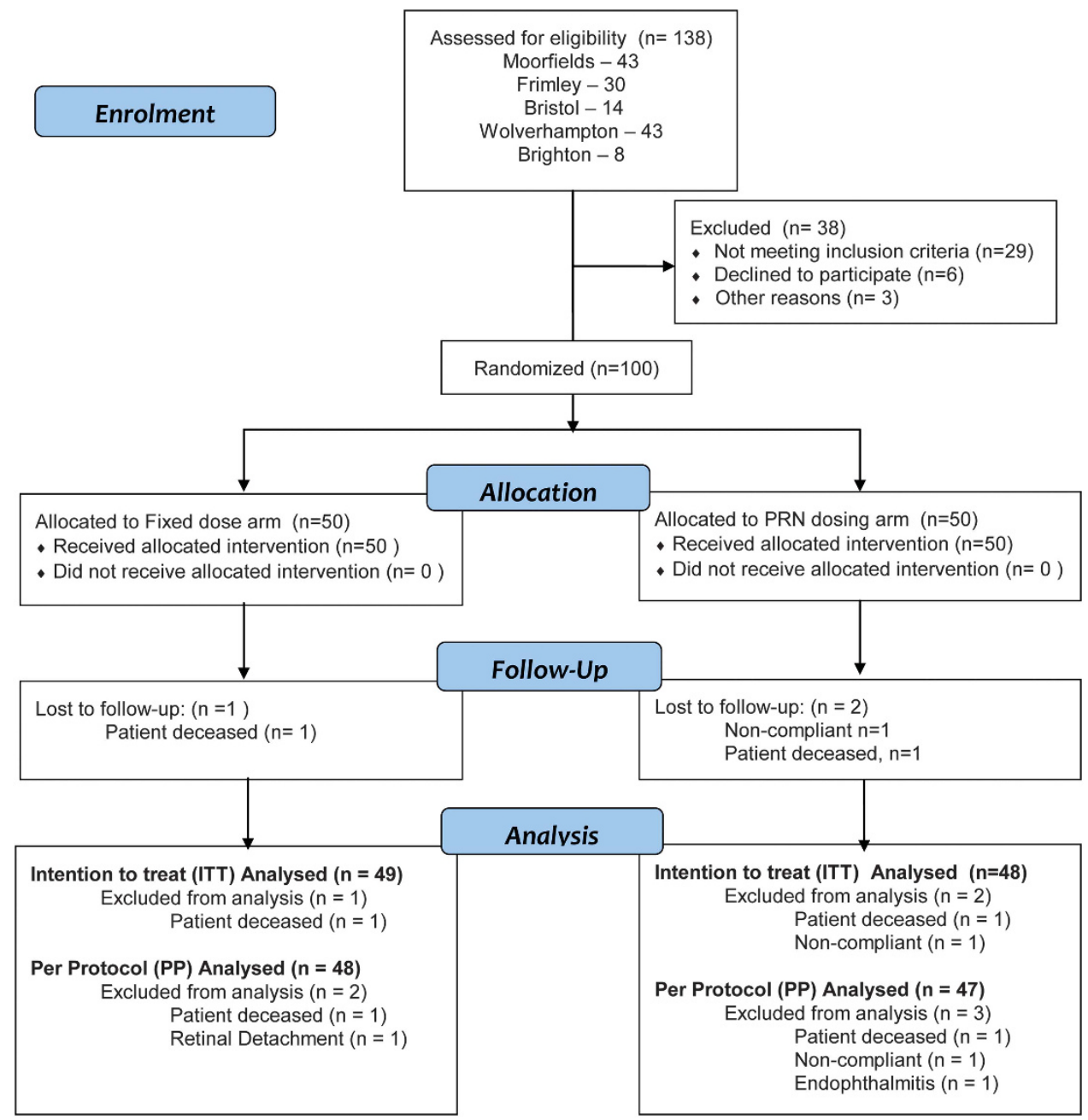

Figure 1 CONSORT flow diagram.

Table 1 Non-ocular baseline characteristics by study arm

\begin{tabular}{|c|c|c|}
\hline & Fixed dosing & PRN dosing \\
\hline Males, $n(\%)(N)$ & $40(80)(50)$ & $34(68)(50)$ \\
\hline Age (years), mean $(\mathrm{SD})(N)$ & $63.8(11.1)(50)$ & $65.4(9.8)(50)$ \\
\hline Ethnicity (N) & $(50)$ & $(50)$ \\
\hline White/Caucasian, $n(\%)$ & $34(68)$ & $35(70)$ \\
\hline Black or African, $n(\%)$ & $5(10)$ & $5(10)$ \\
\hline South Asian, $n(\%)$ & $10(20)$ & $8(16)$ \\
\hline Other, $n(\%)$ & $1(2)$ & $2(4)$ \\
\hline Diabetes $(N)$ & $(50)$ & $(50)$ \\
\hline Type $1, n(\%)$ & $7(14)$ & $2(4)$ \\
\hline Type 2 on insulin, $n(\%)$ & $22(44)$ & $22(44)$ \\
\hline Type 2 on tablets, $n(\%)$ & $21(42)$ & $26(52)$ \\
\hline Duration of diabetes (months), median (IQR) $(N)$ & $192(112,255)(50)$ & $196(124,249)(50)$ \\
\hline HbA1c $(\%)$, mean $(\mathrm{SD})(N)$ & $8.1(1.4)(50)$ & $7.7(1.3)(50)$ \\
\hline Systolic BP (mm Hg), mean (SD) $(N)$ & $148.5(20.5)(50)$ & $142.8(20.5)(50)$ \\
\hline Diastolic BP $(\mathrm{mm} \mathrm{Hg})$, mean $(\mathrm{SD})(N)$ & $79.3(9.8)(50)$ & $77.7(10.8)(50)$ \\
\hline
\end{tabular}

Abbreviations: BP, blood pressure; HbA1c, glycated haemoglobin; IQR, interquartile range; $n$, number of patients; $N$, total number of patients; PRN, pro-re-nata. 
Table 2 Ocular baseline characteristics by study arm

\begin{tabular}{|c|c|c|}
\hline & Fixed dosing & PRN dosing \\
\hline ETDRS BCVA, mean (SD) $(N)$ & $57.5(9.5)(50)$ & $61.2(8.6)(50)$ \\
\hline Duration of DME (months), median (IQR) $(N)$ & $35.5(15.0,51.0)(50)$ & $37.0(18.0,48.0)(50)$ \\
\hline \multicolumn{3}{|l|}{ Prior treatments } \\
\hline Macular laser therapy, $n(\%)(N)$ & $46(92)(50)$ & $48(96)(50)$ \\
\hline Pan-retinal photocoagulation, $n(\%)(N)$ & $14(28)(50)$ & $8(16)(50)$ \\
\hline Intravitreal anti-VEGF, $n(\%)(N)$ & $17(34)(50)$ & $17(34)(50)$ \\
\hline Intravitreal steroids, $n(\%)(N)$ & $5(10)(50)$ & $3(6)(50)$ \\
\hline \multicolumn{3}{|l|}{ OCT findings } \\
\hline CRT $(\mu \mathrm{m})$, mean $(\mathrm{SD})(N)$ & $479.8(128.4)(50)$ & $466.7(144.1)(50)$ \\
\hline $\operatorname{CST}(\mu \mathrm{m})$, mean $(\mathrm{SD})(N)$ & $472.4(113.5)(50)$ & $467.9(126.4)(50)$ \\
\hline Macular volume $\left(\mathrm{mm}^{3}\right)$, mean $(\mathrm{SD})(N)$ & $10.0(2.5)(50)$ & $10.4(2.1)(50)$ \\
\hline \multicolumn{3}{|l|}{ Lens status } \\
\hline Pseudophakic, $n(\%)(N)$ & $16(32)(50)$ & $11(22)(50)$ \\
\hline Phakic, $n(\%)(N)$ & $34(68)(50)$ & $39(78)(50)$ \\
\hline Presence of cataract, $n(\%)(N)$ & $24(70.6)(34)$ & $31(79.5)(39)$ \\
\hline \multicolumn{3}{|l|}{ ETDRS grade of retinopathy } \\
\hline Mild NPDR, $n(\%)(N)$ & $16(32)(50)$ & $17(34)(50)$ \\
\hline Moderate NPDR, $n(\%)(N)$ & $17(34)(50)$ & $21(42)(50)$ \\
\hline Severe NPDR, $n(\%)(N)$ & $5(10)(50)$ & $7(14)(50)$ \\
\hline Treated PDR, $n(\%)(N)$ & $11(22)(50)$ & $5(10)(50)$ \\
\hline Not available, $n(\%)(N)$ & $1(2)(50)$ & $0(0)(50)$ \\
\hline \multicolumn{3}{|l|}{ FFA findings } \\
\hline FAZ GLD (mm), mean (SD) $(N)$ & $808.5(271.8)(50)$ & $769.0(190.4)(50)$ \\
\hline FAZ Area $\left(\mathrm{mm}^{2}\right)$, median (IQR) $(N)$ & $0.5(0.3,0.7)(49)$ & $0.4(0.3,0.6)(50)$ \\
\hline
\end{tabular}

Abbreviations: BCVA, best-corrected visual acuity; CRT, central retinal thickness; CST, central subfield thickness; DME, diabetic macular edema; ETDRS, Early Treatment Diabetic Retinopathy Study; FAZ, foveal avascular zone; FFA, Fundus fluorescein angiography; GLD, greatest linear dimension; IQR, interquartile range; $n$, number of patients; $N$, total number of patients; NPDR, non-proliferative diabetic retinopathy; PDR, proliferative diabetic retinopathy; PRN, pro-re-nata; VEGF, vascular endothelial growth factor.

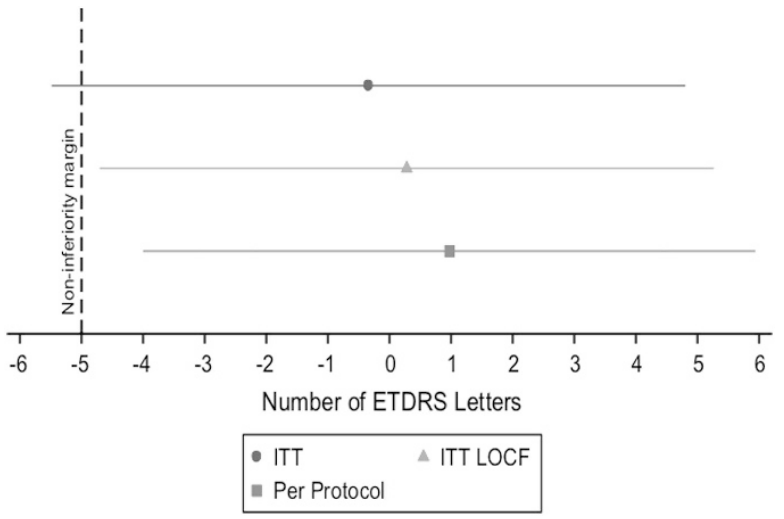

Figure 2 Primary analysis treatment effect estimates with respective to two-sided $90 \%$ confidence interval-fixed $v$ s PRN dosing.

non-inferiority between treatment regimens. Figure 2 summarizes the primary analyses results where the dashed vertical line represents the prespecified noninferiority margin.

\section{Secondary outcomes}

Table 3 shows the outcome of each secondary measure in the two arms.

As a final sensitivity analysis, a within-subgroup analysis of the primary outcome was also performed on patients who were pseudophakic at baseline. The baseline visual acuity of the pseudophakic group was 58.6 in the fixed arm and 61.3 in the PRN arm. The final mean visual acuities of the pseudophakic group in the fixed arm and PRN were 58.3 and 63.2, respectively. Non-inferiority was only observed in the per protocol sensitivity analysis; however, the numbers were small and as such no firm inferences can be drawn.

\section{Safety outcomes}

The proportion of patients who developed IOP $>30 \mathrm{~mm} \mathrm{Hg}$ were $20 \%$ in the fixed arm and $34 \%$ in the PRN arm. Sixtyfour percent $(18 / 28)$ patients initiated on topical IOPlowering medication continued the medication until the end of the study, and three patients required $>1$ topical 
Table 3 Secondary analyses by study arm-efficacy outcome measures at 12 months from baseline ${ }^{\text {a }}$

\begin{tabular}{|c|c|c|c|}
\hline & Fixed dosing & PRN dosing & \\
\hline BCVA (ETDRS letters) & No. of patients, $\mathrm{n}(\%)(\mathrm{N})$ & No. of patients, $\mathrm{n}(\%)(\mathrm{N})$ & Odds ratio \\
\hline \multicolumn{4}{|l|}{ Improvement } \\
\hline$\geq 10$ letters & $12(24)(49)$ & $11(23)(48)$ & $0.82(0.3,2.3)$ \\
\hline$\geq 15$ letters & 7 (14) (49) & $4(8)(48)$ & $1.3(0.33,5.40)$ \\
\hline$\geq 5$ and $<15$ letters & $14(29)(49)$ & $12(25)(48)$ & $1.3(0.50,3.36)$ \\
\hline \multicolumn{4}{|l|}{ Stabilization } \\
\hline$<15$ letters loss & $42(86)(49)$ & $44(92)(48)$ & $0.56(0.15,2.18)$ \\
\hline \multicolumn{4}{|l|}{ No change } \\
\hline$\geq-4$ and $\leq 4$ letters & $17(35)(49)$ & $21(44)(48)$ & $0.7(0.3,1.7)$ \\
\hline \multicolumn{4}{|l|}{ Worsening } \\
\hline$\geq 5$ and $<15$ letters & $4(8)(49)$ & 7 (15) (48) & $0.65(0.17,2.60)$ \\
\hline$\geq 15$ letters & 7 (14) (49) & $4(8)(48)$ & $1.76(0.46,6.76)$ \\
\hline ETDRS grade of retinopathy & No. of patients, $\mathrm{n}(\%)(\mathrm{N})$ & No. of patients, $\mathrm{n}(\%)(\mathrm{N})$ & Odds ratio \\
\hline Mild NPDR & $13(28)(47)$ & $18(40)(45)$ & - \\
\hline Moderate NPDR & $16(34)(47)$ & $16(36)(45)$ & - \\
\hline Severe NPDR & $6(13)(47)$ & $4(9)(45)$ & - \\
\hline Treated PDR & $12(25)(47)$ & 7 (15) (45) & - \\
\hline PROM_composite score change & Mean $(S D)(\mathrm{N})$ & Mean $(S D)(\mathrm{N})$ & Effect estimate $(95 \% \mathrm{CI})$ \\
\hline NEI-VFQ-25 & $3.02(15.4)(49)$ & $-0.45(12.2)(47)$ & $3.1(-2.1,8.3)$ \\
\hline RetDQoL & $-0.38(1.7)(49)$ & $-0.14(1.6)(48)$ & $-0.16(-0.8,0.5)$ \\
\hline RetTSQ & $4.4(12.7)(49)$ & $3.6(15.1)(47)$ & $2.7(-2.3,7.7)$ \\
\hline Central subfield thickness & Mean $(S D)(\mathrm{N})$ & Mean $(S D)(\mathrm{N})$ & Effect estimate $(95 \%$ CI) \\
\hline At 12 months & $292.9(118.9)(47)$ & $372.3(117.3)(47)$ & - \\
\hline Change from baseline & $-179.9(172.4)(47)$ & $-90.1(96.2)(47)$ & $-71.34(-117.33,-25.34)$ \\
\hline Treatment & Mean $(S D) /$ median $(I Q R)(\mathrm{N})$ & Mean $(S D) /$ median $(I Q R)(\mathrm{N})$ & Effect estimate (95\% CI) \\
\hline No. of injections per patient & $2.86(0.45) / 3(3,3)(50)$ & $2.60(0.70) / 3(2,3)(50)$ & $0.26(0.03,0.49)$ \\
\hline
\end{tabular}

Abbreviations: BCVA, best-corrected visual acuity; CI, confidence interval; ETDRS, Early Treatment Diabetic Retinopathy Study; IQR, interquartile range; $n$, number of patients; $N$, total number of patients; NEI-VFQ-25, National Eye Institute Visual Functioning Questionnaire; NPDR, non-proliferative diabetic retinopathy; PDR, proliferative diabetic retinopathy; PRN, pro-re-nata; PROM, patient-related outcome measures; RetDQoL, Retinopathy Dependent Quality of Life questionnaire; RetTSQ, Retinopathy Treatment Satisfaction Questionnaire. ${ }^{\text {a }}$ Adjusted for baseline BCVA and study site.

medication. No patients required surgical intervention for raised IOP in either arm. The topical medications were either initiated at the 8-week visit following a Ozurdex injection or at the next re-treatment visit.

Out of a total of 34 phakic patients in the fixed arm, 27 (79\%) showed new onset or progression of cataract based on change in the LOC II grading by at least 1 grade at the final visit. These included 3 nuclear, 3 cortical, 8 posterior subcapsular cataract (PSCO), and 12 mixed cataract and 1 had cataract surgery. In the PRN arm with 39 phakic patients, $30(77 \%)$ patients showed progression and included 3 nuclear, 6 cortical, 6 PSCO, and 11 mixed cataract and 4 had cataract surgery. There was one case of retinal detachment in the PRN arm and one case of endophthalmitis in the fixed arm, and both events were reported as related to the intervention.

The change in greatest linear dimension and area of foveal vascular zone from baseline to 12 months were not significantly different between arms. There was no difference between arms in changes in systolic and diastolic blood pressure and glycated haemoglobin. The mean $\mathrm{HbA} 1 \mathrm{C}$ in the fixed arm at exit was 8.1 (SD 1.6), and in the PRN group, it was 7.8 (SD 1.5).

\section{Discussion}

The ITT (available case) analysis did not demonstrate non-inferiority. However, the per protocol and the 
post-hoc ITT analysis supported non-inferiority. We also conducted a post-hoc sensitivity ITT analysis using LOCF for the three subjects who withdrew. This agreed with the PP population, and further it should be noted that the original ITT analysis missed the margin by only half a letter. In summary, therefore, we believe that this study lends support to the statement of non-inferiority, that is, the results of this trial show that there is no evidence that 5-monthly fixed dosing of Ozurdex is non-inferior to OCT-guided PRN regimen of Ozurdex in patients with refractory DMO in terms of visual acuity at 12 months. Both arms showed similar visual acuity changes despite more frequent monitoring in the PRN arm. Likewise, both arms showed low mean change in visual acuity at 12 months from baseline despite significant reduction in the central macular thickness, more so in the fixed arm.

About one in five patients also lost $\geq 5$ letters with Ozurdex in both arms, and this concurs with previous studies. In the BEVORDEX study, 11\% lost $\geq 10$ letters in the Ozurdex arm compared with none in the bevacizumab arm at 12 months. Most anti-VEGF trials report $<5 \%$ of patients losing vision. This may be attributed mainly owing to the development and progression of cataract.

The ocular and systemic safety profiles of Ozurdex in both treatment groups of this study were very similar to previous reports with no unexpected events. Although cataract progression and IOP increases are expected complications of corticosteroid treatment, the incidence did not differ between treatment pathways in this study. The increases in IOP that occurred were typically manageable with topical medication. No patients required surgical management of IOP. It should be noted that the summary of product characteristics of Ozurdex states that the drug is contraindicated in advanced glaucoma, which cannot be adequately controlled by medicinal products alone.

The results of this study suggest that patients need not be reviewed for IOP check at 1 week following Ozurdex injection as no patients developed a rise in IOP at this time point. In most patients who developed an IOP rise, this was observed at the visit of 8 weeks after injection. We therefore recommend a postinjection IOP check at about 4-8 weeks, especially in eyes with established glaucoma or ocular hypertension or previous history of steroid-induced ocular hypertension in both arms.

As previously shown, cataract progression is dose related and more frequent dosing than 6 monthly resulted in a higher proportion of cataract development and progression that affected final visual acuity gain. It should be noted that there is no standard definition of progression of cataract or for the threshold for cataract surgery. Differences in rate of cataract progression reported between studies using varying dosing regimens may not be related to the dosing regimen. We defined cataract progression as a 1-step change in LOC
II score while the BEVORDEX study defined as a 2-step change in LOC II grading.

We believe that intravitreal Ozurdex is very effective in causing resolution of macular fluid. However, unlike the earlier studies such as the MEAD study that included patients with persistent fluid postlaser treatment, recent studies include patients who have been refractory to laser therapy and anti-VEGF agents. Therefore, these are truly refractory cases and visual acuity is unlikely to improve in many of these cases despite complete resolution of macular oedema.

If Ozurdex is planned as an alternate option for patients with refractory DMO, this study suggests that 5-monthly fixed dosing is an effective approach and may be more acceptable to patients. Patients should be warned about cataract progression and that significant gains in visual acuity is less likely compared with anti-VEGF agents as Ozurdex is used a second-line agents in pseudophakic eyes with refractory DMO only.

The strengths of this trial include secure randomisation, size, multicentre design, low rates of losses to follow-up, and use of outcome measures appropriate to the primary outcome. Limitations of the study include the fact that the 12-month cutoff of the study may have been more advantageous to the fixed arm than the PRN arm because all patients received mandated dosing in the fixed arm at 10 months and the maximal effect on vision and macular thickness is expected at 12 months while the injections flexibility in the PRN arm may have meant that not all patients would have attained maximal efficacy by 12 months. However, this did not alter the visual outcome between arms and may only explain the differences in central macular thickness between arms.

To our knowledge, this is the first large prospective, randomized controlled trial of dosing regimens with Ozurdex in DMO. The results of this study provide concise data suggesting that 5-monthly fixed dosing is non-inferior to OCT-guided PRN treatment both in terms of visual outcome and safety profile.

\section{Summary}

What was known before

- Ozurdex is recommended for refractory diabetic macular oedema.

What this study adds

- Five-monthly fixed dosing of Ozurdex is non-inferior to OCT-guided PRN dosing in refractory diabetic macular oedema.

\section{Conflict of interest}

YY: consultant-Bayer Healthcare, Alimera Sciences; personal fees-Alcon Inc., Pfizer Inc., Novartis 
Pharmaceutical, Inc., Allergan Inc., Bayer Healthcare; lecture fees-Bayer Healthcare, Novartis

Pharmacueticals, Plc; grant outside the submitted work: Bayer Healthcare, Novartis Pharmaceutical, Inc., Roche Inc.; NN: consultant-Novartis Pharmacuetical, Inc. GM: consultant-Bayer Healthcare, Alcon Inc., Novartis Pharmaceutical, Inc., Allergan Inc.; grant outside the submitted work-Bayer Healthcare; Novartis Pharmaceutical, Inc., Allergan Inc., Alcon Plc, Roche Inc. CB: consultant-Bayer Healthcare, Alimera Sciences, Alcon Inc., Novartis Pharmaceutical, Inc., Allergan Inc.; lecture fees-Bayer Healthcare, Novartis Pharmacueticals, Plc; grant outside the submitted workBayer Healthcare; Novartis Pharmaceutical, Inc., Allergan Inc., Alcon Plc, Roche Inc. PH: consultant-Bayer Healthcare, Novartis Pharmaceutical, Inc., Allergan Inc.; lecture fees-Bayer Healthcare, Novartis

Pharmacueticals, Plc; grant outside the submitted workBayer Healthcare; Novartis Pharmaceutical, Inc., Allergan Inc. SS: consultant-Bayer Healthcare, Novartis Pharmaceutical, Inc., Allergan Inc., Roche Inc.; lecturerBayer Healthcare, Novartis Pharmacueticals, Plc, Allergan Plc; grant outside the submitted work-Bayer Healthcare; Novartis Pharmaceutical, Inc., Allergan Inc., Roche Inc. The other authors declare no conflict of interest.

\section{Acknowledgements}

The study was funded (including drug supply) by an Investigator Initiated Grant from Allergan Pharmaceuticals Ireland, Grant No: ISS.RET.022. The funding organization had no role in the design or conduct of this research. The funding was paid to all the institutions of the authors. The research was supported by the National Institute for Health Research (NIHR) Biomedical Research Centre based at Moorfields Eye Hospital NHS Foundation Trust and UCL Institute of Ophthalmology. The views expressed are those of the authors and not necessarily those of the NHS, the NIHR, or the Department of Health. The Randomization system and the MACRO database was supported by the United Kingdom Clinical Research Collaboration-registered King's Clinical Trials Unit at King'sHealth Partners, which is part funded by the NIHR Biomedical Research Centre for Mental Health at South London and Maudsley NHS Foundation Trust and King's College London and the NIHR Evaluation, Trials and Studies Coordinating Centre. Chair of TSC: Sheena Koshy FRCOphth; DMC members: Jignesh Patel, FRCOphth, Niaz Islam, FRCOphth, Irene Stratton, MSc.

\section{References}

1 Wenick AS, Bressler NM. Diabetic macular edema: current and emerging therapies. Middle East Afr J Ophthalmol 2012; 19(1): 4-12.
2 Mitchell P, Bandello F, Schmidt-Erfurth U, Lang GE, Massin P, Schlingemann $\mathrm{RO}$ et al. The RESTORE study: ranibizumab monotherapy or combined with laser versus laser monotherapy for diabetic macular edema. Ophthalmology 2011; 118: 615-625.

3 Elman MJ, Bressler NM, Qin H, Beck RW, Ferris III FL, Friedman SM et al. Expanded 2-year follow-up of ranibizumab plus prompt or deferred laser or triamcinolone plus prompt laser for diabetic macular edema. Ophthalmology 2011; 118: 609-614.

4 Bressler SB, Qin H, Beck RW, Chalam KV, Kim JE, Melia M et al. Factors associated with changes in visual acuity and central subfield thickness at 1 year after treatment for diabetic macular edema with ranibizumab. Arch Ophthalmol 2012; 130: 1153-1161.

5 Sivaprasad S, Crosby-Nwaobi R, Esposti SD, Peto T, Rajendram R, Michaelides $M$ et al. Structural and functional measures of efficacy in response to bevacizumab monotherapy in diabetic macular oedema: exploratory analyses of the BOLT study (report 4). PLoS One 2013; 8(8): e72755.

6 Bandello F, Cunha-Vaz J, Chong NV, Lang GE, Massin P, Mitchell $\mathrm{P}$ et al. New approaches for the treatment of diabetic macular oedema: recommendations by an expert panel. Eye (Lond) 2012; 26(4): 485-493.

7 Diabetic Retinopathy Clinical Research Network (DRCR. net), Beck RW, Edwards AR, Aiello LP, Bressler NM, Ferris F et al. Three-year follow-up of a randomized trial comparing focal/grid photocoagulation and intravitreal triamcinolone for diabetic macular edema. Arch Ophthalmol 2009; 127(3): 245-251.

8 Stewart MW. Corticosteroid use for diabetic macular edema: old fad or new trend? Curr Diab Rep 2012; 12(4): 364-375.

9 Boyer DS, Yoon YH, Belfort Jr R, Bandello F, Maturi RK, Augustin AJ et al. Three-year, randomized, sham-controlled trial of dexamethasone intravitreal implant in patients with diabetic macular edema. Ophthalmology 2014; 121(10): 1904-1914.

10 Callanan DG, Gupta S, Boyer DS, Ciulla TA, Singer MA, Kuppermann BD et al. Dexamethasone intravitreal implant in combination with laser photocoagulation for the treatment of diffuse diabetic macular edema. Ophthalmology 2013; 120(9): 1843-1851.

11 Mathew R, Pearce E, Muniraju R, Abdel-Hay A, Sivaprasad S. Monthly OCT monitoring of Ozurdex for macular oedema related to retinal vascular diseases: re-treatment strategy (OCTOME Report 1). Eye (Lond) 2014; 28(3): 318-326.

12 Gillies MC, Lim LL, Campain A, Quin GJ, Salem W, Li J et al. A randomized clinical trial of intravitreal bevacizumab versus intravitreal dexamethasone for diabetic macular edema: the BEVORDEX study. Ophthalmology 2014; 121(12): 2473-2481.

13 Mangione CM, Lee PP, Gutierrez PR, Spritzer K, Berry S, Hays RD et al. Development of the 25-item National Eye Institute Visual Function Questionnaire. Arch Ophthalmol 2001; 119(7): 1050-1058.

14 Brose LS, Bradley C. Psychometric development of the retinopathy treatment satisfaction questionnaire (RetTSQ). Psychol Health Med 2009; 14(6): 740-754.

15 Brose LS, Bradley C. Psychometric development of the individualized Retinopathy-Dependent Quality of Life Questionnaire (RetDQoL). Value Health 2010; 13(1): 119-127.

16 Wilkinson CP, Ferris 3rd FL, Klein RE, Lee PP, Agardh CD, Davis $\mathrm{M}$ et al. Proposed international clinical diabetic 
retinopathy and diabetic macular edema disease severity scales. Ophthalmology 2003; 110(9): 1677-1682.

17 Chylack Jr LT , Leske MC, Sperduto R, Khu P, McCarthy D. Arch Ophthalmol 1988; 106(3): 330-334.

18 Massin P, Bandello F, Garweg JG, Hansen LL, Harding SP, Larsen $\mathrm{M}$ et al. Safety and efficacy of ranibizumab in diabetic macular edema (RESOLVE Study): a 12-month, randomized, controlled, double-masked, multicenter phase II study. Diabetes Care 2010; 33(11): 2399-2405.

19 CATT Research Group, Martin DF, Maguire MG, Ying GS, Grunwald JE, Fine SL et al. Ranibizumab and bevacizumab for neovascular age-related macular degeneration. $N$ Engl J Med 2011; 364(20): 1897-1908. 